\title{
On a co-universal arrow in the construct of $n$-ary hyperalgebras
}

\author{
Josef Šlapal
}




\title{
ON A CO-UNIVERSAL ARROW IN THE CONSTRUCT OF $n$-ARY HYPERALGEBRAS
}

\author{
J. ŠLAPAL \\ Received 23 October, 2013
}

\begin{abstract}
We introduce and study a new operation of product of $n$-ary hyperalgebras which lies, with respect to set inclusion, between their cartesian product and the cartesian product of their idempotent hulls. For every fixed $n$-ary hyperalgebra, the product introduced gives an endofunctor of the construct of $n$-ary hyperalgebras. We define a power of $n$-ary hyperalgebras and specify a class of $n$-ary hyperalgebras such that, with respect to the endofunctor, the power together with the evaluation map constitute a co-universal arrow for each hyperalgebra of the class.
\end{abstract}

2010 Mathematics Subject Classification: 08B25; $08 \mathrm{C} 05$

Keywords: hyperalgebra, diagonality, mediality, combined product, co-universal arrow with respect to a functor

\section{INTRODUCTION}

Hyperalgebras proved to be useful generalizations of algebras having many application in various branches of mathematics and computer science (automata theory). This lead to a rapid development of the theory of hyperalgebras since the beginning of 90's of the last century - see e.g. [3]. The aim of this paper is to contribute to the development.

In his pioneering paper [2], G. Birkhoff introduced the cardinal (i.e., direct) arithmetic of partially ordered sets and showed that it behaves analogously to the arithmetic of natural numbers. Several authors then extended the cardinal arithmetic to relational systems - see e.g. [7,8] and [10,11]. Conversely, the cardinal arithmetic has been restricted from relational systems to $n$-ary and universal algebras in [13] and [12], respectively. Finally, in [14], the cardinal arithmetic was extended from universal algebras to universal hyperalgebras.

In [14], an operation of a power of hyperalgebras (of the same type) was introduced and studied. The results of [14] determine a class of hyperalgebras such that, with respect to the endofunctor of the construct of hyperalgebras (of the same type) given by

This work was supported by the project NETME+ (LO1202) cofunded by the Ministry of Education of the Czech Republic under the National Sustainability Programme I. 
the cartesian product and a fixed idempotent hyperalgebra, the power together with the evaluation map constitute a co-universal arrow for each hyperalgebra of the class. In the present note, we restrict our considerations to $n$-ary hyperalgebras, i.e., hyperalgebras with just one $n$-ary hyperoperation. For $n$-ary hyperalgebras, we introduce an operation of product which is obtained as the restriction of the product of relational systems introduced and studied in [8]. The operation lies, with respect to set inclusion, between the cartesian product of $n$-ary hyperalgebras and the cartesian product of their idempotent hulls. For idempotent hyperalgebras, the cartesian product and the introduced one coincide. We will show that the above mentioned class has the property that, with respect to the endofunctor of the construct of $n$-ary hyperalgebras given by the new product and arbitrary (not only idempotent) but fixed $n$-ary hyperalgebra, the power together with the evaluation map constitute a co-universal arrow for each hyperalgebra of the class. We then get the first exponential law with respect to the new product which, unlike the first exponential law with respect to the cartesian product, is valid for arbitrary $n$-ary algebras in the exponents, not only for the idempotent ones.

\section{COMBINED PRODUCT OF HYPERALGEBRAS}

For the basic concepts used concerning $n$-ary hyperalgebras we refer to [2] and for those concerning category theory we refer to [1]. Let $n$ be a nonnegative integer. By an $n$-ary hyperalgebra we understand a pair $\mathbf{G}=(G, p)$ where $G$ is a set, the so-called carrier of $\mathbf{G}$, and $p: G^{n} \rightarrow \exp G \backslash\{\varnothing\}$ is a map, the so-called $n$-ary hyperoperation of $\mathbf{G}$. To avoid some nonwanted singularities, only the $n$-ary hyperalgebras with $n>0$ are considered in this note. An $n$-ary hyperalgebra $\mathbf{G}=(G, p)$ with the property card $p\left(x_{1}, \ldots, x_{n}\right)=1$ for every $x_{1}, \ldots, x_{n} \in G$ is called an $n$-ary algebra. Binary hyperalgebras are usually called hypergroupiods.

Let $\mathbf{H}=(H, q), \mathbf{G}=(G, p)$ be a pair of $n$-ary hyperalgebras. A map $f: H \rightarrow G$ is called a homomorphism of $\mathbf{H}$ into $\mathbf{G}$ if $f\left(q\left(x_{1}, \ldots, x_{n}\right)\right) \subseteq p\left(f\left(x_{1}\right), \ldots, f\left(x_{n}\right)\right)$. The set of all homomorphisms of $\mathbf{H}$ into $\mathbf{G}$ will be denoted by $\operatorname{Hom}(\mathbf{H}, \mathbf{G})$. We denote by $H Y P_{n}$ the construct of $n$-ary hyperalgebras with homomorphisms as morphisms. Subobjects in $H Y P_{n}$ are called subhyperalgebras. Clearly, $\mathbf{G}$ is a subhyperalgebra of $\mathbf{H}$ if $G \subseteq H$ and $p\left(x_{1}, \ldots, x_{n}\right)=q\left(x_{1}, \ldots, x_{n}\right)$, whenever $x_{1}, \ldots x_{n} \in H$. We write $\mathbf{G} \cong \mathbf{H}$ if $\mathbf{G}$ and $\mathbf{H}$ are isomorphic in $H Y P_{n}$ and we write $\mathbf{G} \preceq \mathbf{H}$ if $\mathbf{G}$ may be embedded into $\mathbf{H}$ in $H Y P_{n}$, i.e., if there exists a subalgebra $\mathbf{H}^{\prime}$ of $\mathbf{H}$ such that $\mathbf{G} \cong \mathbf{H}^{\prime}$.

Given $n$-ary hyperalgebras $\mathbf{G}=(G, p)$ and $\mathbf{H}=(G, q)$, we put $\mathbf{G} \leq \mathbf{H}$ if $p\left(x_{1}, \ldots, x_{n}\right) \subseteq q\left(x_{i}, \ldots, x_{n}\right)$ for every $x_{1}, \ldots, x_{n} \in G$.

If $p$ is an $n$-ary hyperoperation on a set $G$ and $X_{1}, \ldots, X_{n}$ are nonempty subsets of $G$, we put $p\left(X_{1}, \ldots, X_{n}\right)=\bigcup\left\{p\left(x_{1}, \ldots, x_{n}\right) ; x_{1} \in X_{1}, \ldots, x_{n} \in X_{n}\right\}$.

Let $\mathbf{G}_{i}=\left(G_{i}, p_{i}\right), i \in I$, be a family of $n$-ary hyperalgebras. We denote by $\prod_{i \in I} \mathbf{G}_{i}$ the cartesian product of the family in $H Y P_{n}$. Thus, $\prod_{i \in I} \mathbf{G}_{i}$ 
$=\left(\prod_{i \in I} G_{i}, p\right)$ is the $n$-ary hyperalgebra where $p\left(f_{1}, \ldots, f_{n}\right)=\prod_{i \in I} p_{i}\left(f_{1}(i), \ldots\right.$, $\left.f_{n}(i)\right)$ whenever $f_{1}, \ldots, f_{n} \in \prod_{i \in I} G_{i}$. As usual, if the set $I$ is finite, say $I=$ $\{1, \ldots, m\}$, then we write $\mathbf{G}_{1} \times \ldots \times \mathbf{G}_{m}$ instead of $\prod_{i \in I} \mathbf{G}_{i}$. Further, we define the direct sum of a family $\mathbf{G}_{i}=\left(G, p_{i}\right), i \in I$, of $n$-ary hyperalgebras to be the $n$ ary hyperalgebra $\sum_{i \in I} \mathbf{G}_{i}=(G, q)$ where, for every $x_{1}, \ldots, x_{n} \in G, q\left(x_{1}, \ldots, x_{n}\right)=$ $\bigcup_{i \in I} p_{i}\left(x_{1}, \ldots, x_{n}\right)$. If the set $I$ is finite, say $I=\{1, \ldots, m\}$, then we write $\mathbf{G}_{1} \uplus$ $\ldots \uplus \mathbf{G}_{m}$ instead of $\sum_{i \in I} \mathbf{G}_{i}$.

An $n$-ary hyperalgebra $\mathbf{G}=(G, p)$ is said to be idempotent if, for every $x \in G, x \in$ $p\left(x_{1}, \ldots, x_{n}\right)$ whenever $x=x_{1}=\ldots=x_{n}$. Let $\mathbf{G}=(G, p)$ be an $n$-ary hyperalgebra. For every $x_{1}, \ldots, x_{n} \in G$, we put

$$
\bar{p}\left(x_{1}, \ldots, x_{n}\right)= \begin{cases}p\left(x_{1}, \ldots, x_{n}\right) \cup\left\{x_{1}\right\} & \text { if } x_{1}=\ldots=x_{n}, \\ p\left(x_{1}, \ldots, x_{n}\right) & \text { if } x_{i} \neq x_{j} \text { for some } i, j \in\{1, \ldots, n\} .\end{cases}
$$

The $n$-ary hyperalgebra $(G, \bar{p})$ is called the idempotent hull of $\mathbf{G}$ and is denoted by $\overline{\mathbf{G}}$.

Let $\mathbf{G}_{i}=\left(G_{i}, p_{i}\right), i \in I$, be a non-empty family of $n$-ary hyperalgebras. The combined product of the family is the $n$-ary hyperalgebra $\bigotimes_{i \in I} \mathbf{G}_{i}=\sum_{i \in I} \prod_{j \in I} \mathbf{G}_{i j}$ where

$$
\mathbf{G}_{i j}= \begin{cases}\overline{\mathbf{G}}_{j} & \text { if } i=j, \\ \mathbf{G}_{j} & \text { if } i \neq j .\end{cases}
$$

Thus, $\bigotimes_{i \in I} \mathbf{G}_{i}=\left(\prod_{i \in I} G_{i}, r\right)$ where, for any $f, f_{1}, \ldots, f_{n} \in \prod_{i \in I} G_{i}$, $f \in r\left(f_{1}, \ldots, f_{n}\right)$ if and only if there exists a subset $J \subseteq I$, card $J \leq 1$, such that $f(i) \in p_{i}\left(f_{1}(i), \ldots, f_{n}(i)\right)$ for every $i \in I \backslash J$ and $f(i)=f_{1}(i)=\ldots=f_{n}(i)$ for every $i \in J$.

If the set $I$ is finite, say $I=\{1, \ldots, m\}$, we write $\mathbf{G}_{1} \otimes \ldots \otimes \mathbf{G}_{m}$ instead of $\bigotimes_{i \in I} G_{i}$. We then clearly have $\mathbf{G}_{1} \otimes \ldots \otimes \mathbf{G}_{m}=\left(\mathbf{G}_{11} \times \mathbf{G}_{12} \times \ldots \times \mathbf{G}_{1 m}\right) \uplus\left(\mathbf{G}_{21} \times\right.$ $\left.\mathbf{G}_{22} \times \ldots \times \mathbf{G}_{2 m}\right) \uplus \ldots \uplus\left(\mathbf{G}_{m 1} \times \mathbf{G}_{m 2} \ldots, \times \mathbf{G}_{m m}\right)=\left(\overline{\mathbf{G}}_{1} \times \mathbf{G}_{2} \times \ldots \times \mathbf{G}_{m}\right) \uplus\left(\mathbf{G}_{1} \times \overline{\mathbf{G}}_{2}\right.$ $\left.\times \ldots \times \mathbf{G}_{m}\right) \uplus \ldots \uplus\left(\mathbf{G}_{1} \times \mathbf{G}_{2} \times \ldots \times \mathbf{G}_{m}\right)$.

In particular, if $I=\{1,2\}$, then, for any $\left(x_{1}, y_{1}\right), \ldots,\left(x_{n}, y_{n}\right) \in \mathbf{G}_{1} \times \mathbf{G}_{2},(x, y) \in$ $r\left(\left(x_{1}, y_{1}\right), \ldots,\left(x_{n}, y_{n}\right)\right)$ if and only if one of the following three conditions is satisfied:

(i) $x \in p_{1}\left(x_{1}, \ldots, x_{n}\right)$ and $y \in p_{2}\left(y_{1}, \ldots, y_{n}\right)$,

(ii) $x=x_{1}=x_{2}=\ldots=x_{n}$ and $y \in p_{2}\left(y_{1}, \ldots, y_{n}\right)$,

(iii) $x \in p_{1}\left(x_{1}, \ldots, x_{n}\right)$ and $y=y_{1}=y_{2}=\ldots=y_{n}$.

If card $I=1$, say $I=\{i\}$, then $\bigotimes_{i \in I} \mathbf{G}_{i}=\overline{\mathbf{G}}_{i}$.

For every $n$-ary hyperalgebra $\mathbf{H}=(H, q)$, the operation of combined product defines an endofunctor $\mathbf{H} \otimes-: H Y P_{n} \rightarrow H Y P_{n}$ which assigns to every morphism $f: \mathbf{G}_{1} \rightarrow \mathbf{G}_{2}$ in $H Y P_{n}$ the morphism $i d_{H} \times f: \mathbf{H} \otimes \mathbf{G}_{1} \rightarrow \mathbf{H} \otimes \mathbf{G}_{2}$. This endofunctor will be studied in the next section.

Remark 1. Let $\mathbf{G}_{i}, i \in I$, be a non-empty family of $n$-ary hyperalgebras. We clearly have $\prod_{i \in I} \mathbf{G}_{i} \leq \bigotimes_{i \in I} \mathbf{G}_{i} \leq \prod_{i \in I} \overline{\mathbf{G}}_{i}=\bigotimes_{i \in I} \overline{\mathbf{G}}_{i}$ and $\overline{\bigotimes_{i \in I} \mathbf{G}_{i}} \leq \bigotimes_{i \in I} \overline{\mathbf{G}}_{i}$. 
If card $I \leq 2$, then $\overline{\bigotimes_{i \in I} \mathbf{G}_{i}}=\prod_{i \in I} \overline{\mathbf{G}}_{i}$. If $\mathbf{G}_{i}$ is idempotent for every $i \in I$, then all the previous inequalities become equalities. If at most one of the $n$-ary hyperalgebras $\mathbf{G}_{i}, i \in I$, is not idempotent, then $\bigotimes_{i \in I} \mathbf{G}_{i}$ is idempotent.

Theorem 1. Let $\mathbf{G}_{i}=\left(G_{i}, p_{i}\right), i \in I \neq \varnothing$, and $\mathbf{H}=(H, q)$ be n-ary hyperalgebras. Then $\sum_{i \in I} \mathbf{H} \otimes \mathbf{G}_{i}=\mathbf{H} \otimes \sum_{i \in I} \mathbf{G}_{i}$.

Proof. Let $\sum_{i \in I} \mathbf{G}_{i}=(G, s), \mathbf{H} \otimes \mathbf{G}_{i}=\left(H \times G, r_{i}\right)$ for each $i \in I, \mathbf{H} \otimes \sum_{i \in I} \mathbf{G}_{i}$ $=(H \times G, u)$ and $\sum_{i \in I} \mathbf{H} \otimes \mathbf{G}_{i}=(H \times G, v)$. We will show that $u\left(\left(x_{1}, y_{1}\right), \ldots,\left(x_{n}, y_{n}\right)\right)=v\left(\left(x_{1}, y_{1}\right), \ldots,\left(x_{n}, y_{n}\right)\right)$ for every $\left(x_{1}, y_{1}\right), \ldots,\left(x_{n}, y_{n}\right) \in$ $H \times G$. It is easy to see that the following five conditions are equivalent:

(a) $(x, y) \in u\left(\left(x_{1}, y_{1}\right), \ldots,\left(x_{n}, y_{n}\right)\right)$;

(b) one of the following three cases occurs:

(i) $x \in q\left(x_{1}, \ldots, x_{n}\right)$ and $y \in s\left(y_{1}, \ldots, y_{n}\right)$,

(ii) $x=x_{1}=x_{2}=\ldots=x_{n}$ and $y \in s\left(y_{1}, \ldots, y_{n}\right)$,

(iii) $x \in q\left(x_{1}, \ldots, x_{n}\right)$ and $y=y_{1}=y_{2}=\ldots=y_{n}$;

(c) one of the following three cases occurs:

(i) $x \in q\left(x_{1}, \ldots, x_{n}\right)$ and $y \in p_{i}\left(y_{1}, \ldots, y_{n}\right)$ for some $i \in I$,

(ii) $x=x_{1}=x_{2}=\ldots=x_{n}$ and $y \in p_{i}\left(y_{1}, \ldots, y_{n}\right)$ for some $i \in I$,

(iii) $x \in q\left(x_{1}, \ldots, x_{n}\right)$ and $y=y_{1}=y_{2}=\ldots=y_{n}$;

(d) $(x, y) \in r_{i}\left(\left(x_{1}, y_{1}\right), \ldots,\left(x_{n}, y_{n}\right)\right)$ for some $i \in I$;

(e) $(x, y) \in v\left(\left(x_{1}, y_{1}\right), \ldots,\left(x_{n}, y_{n}\right)\right)$.

This proves the statement.

Definition 1. An $n$-ary hyperalgebra $(G, p)$ is called medial if, for every $n \times$ $n$-matrix $\left(a_{i j}\right)$ over $G$, from $x_{i} \in p\left(a_{i 1}, \ldots, a_{i n}\right)$ for each $i=1, \ldots, n$ and $y_{j} \in$ $p\left(a_{1 j}, \ldots, a_{n j}\right)$ for each $j=1, \ldots, n$ it follows that $p\left(x_{1}, \ldots, x_{n}\right)=p\left(y_{1}, \ldots, y_{n}\right)$.

The medial $n$-ary algebras are studied in [13]. The mediality may be extended, in a natural way, from $n$-ary algebras onto universal algebras. The medial universal algebras are often called commutative and they were dealt with e.g. in [5,6] and [12]. The medial groupoids are studied in [4].

Example 1. (1) Let $(X, \leq)$ be a partially ordered set with a least element 0 and let $A$ be the set of all atoms of $(X, \leq)$. Put $0^{\prime}=\{0\}$ for any $x \in X$ with $x \neq 0$ put $x^{\prime}=\{y \in X ; y<x$ and $y \in A \cup\{0\}\}$. Further, for any pair $x, y \in X$ put $x * y=$ $x^{\prime} \cap y^{\prime}$. Then $(X, *)$ is a medial hypergroupoid. Indeed, it can easily be seen that for any $a, b, c, d \in X$ we have $x * y=f * g$ whenever $x \in a * b, y \in c * d, f \in a * c$ and $g \in b * d$.

(2) Every unary algebra $\mathbf{G}$ consisting of two-element cycles is medial and, moreover, the unary hyperalgebra $\overline{\mathbf{G}}$ is medial, too.

It may easily be seen that the cartesian product of a family of medial $n$-ary hyperalgebras is a medial $n$-ary hyperalgebra. But this is not generally true for the combined 
product of $n$-ary hyperalgebras. With respect to the combined product, we have the following result:

Proposition 1. Let $\mathbf{G}, \mathbf{H}$ be $n$-ary hyperalgebras. If $\overline{\mathbf{G}}, \overline{\mathbf{H}}$ are medial, then so is $\overline{\mathbf{G} \otimes \mathbf{H}}$.

Proof. Since the cartesian product of a family of medial $n$-ary hyperalgebras is a medial $n$-ary hyperalgebra, the statement follows from the obvious equality $\overline{\mathbf{G} \otimes \mathbf{H}}=$ $\overline{\mathbf{G}} \times \overline{\mathbf{H}}$ (see Remark 1).

\section{POWER OF HYPERALGEBRAS}

Lemma 1. Let $\mathbf{H}=(H, q)$ and $\mathbf{G}=(G, p)$ be $n$-ary hyperalgebras and let $f_{1}, \ldots, f_{n} \in \operatorname{Hom}(\mathbf{H}, \mathbf{G})$. Let $f: H \rightarrow G$ be a map such that $f(x) \in$ $p\left(f_{1}(x), \ldots, f_{n}(x)\right)$ for every $x \in H$. If $\mathbf{G}$ is medial, then $f$ is a homomorphism from $\mathbf{H}$ into $\mathbf{G}$.

Proof. Let $x_{1}, \ldots, x_{n} \in H$. We will show that $f\left(q\left(x_{1}, \ldots, x_{n}\right)\right) \subseteq p\left(f\left(x_{1}\right), \ldots\right.$, $\left.f\left(x_{n}\right)\right)$. Let $y \in f\left(q\left(x_{1}, \ldots, x_{n}\right)\right)$. Then there exists $x \in q\left(x_{1}, \ldots, x_{n}\right)$ such that $y=f(x)$, so $f(x) \in f\left(q\left(x_{1}, \ldots, x_{n}\right)\right)$. Since $f_{1}, \ldots, f_{n} \in \operatorname{Hom}(\mathbf{H}, \mathbf{G})$ and $x \in$ $q\left(x_{1}, \ldots, x_{n}\right)$, we have $f_{j}(x) \in f_{j}\left(q\left(x_{1}, \ldots, x_{n}\right)\right) \subseteq p\left(f_{j}\left(x_{1}\right), \ldots, f_{j}\left(x_{n}\right)\right)$ for each $j=1, \ldots, n$. By the assumption, we have $f\left(x_{i}\right) \in p\left(f_{1}\left(x_{i}\right), \ldots, f_{n}\left(x_{i}\right)\right)$ for each $i=1, \ldots, n$ and $f(x) \in p\left(f_{1}(x), \ldots, f_{n}(x)\right)$. Since $\mathbf{G}$ is medial, $p\left(f_{1}(x), \ldots, f_{n}(x)\right)$ $=p\left(f\left(x_{1}\right), \ldots, f\left(x_{n}\right)\right)$. Thus, $y=f(x) \in p\left(f\left(x_{1}\right), \ldots, f\left(x_{n}\right)\right)$.

Given sets $G, H$, we denote by $G^{H}$ the set of all maps of $H$ into $G$. The bijection $\varphi:\left(G^{H}\right)^{K} \rightarrow G^{H \times K}$ (where $G, H, K$ are sets and $\times$ denotes the cartesian product) given by $\varphi(h)(x, y)=h(y)(x)$ whenever $h \in\left(G^{H}\right)^{K}, x \in H$ and $y \in K$ will be called canonical.

Definition 2. Let $\mathbf{H}=(H, q)$ and $\mathbf{G}=(G, p)$ be $n$-ary hyperalgebras and let $\mathbf{G}$ be medial. The power of $\mathbf{G}$ and $\mathbf{H}$ is the $n$-ary hyperalgebra $\mathbf{G}^{\mathbf{H}}=(\operatorname{Hom}(\mathbf{H}, \mathbf{G}), r)$ where, for any $f_{1}, \ldots, f_{n} \in \operatorname{Hom}(\mathbf{H}, \mathbf{G}), \quad r\left(f_{1}, \ldots, f_{n}\right)=\left\{f \in G^{H} ; f(x) \in\right.$ $p\left(f_{1}(x), \ldots, f_{n}(x)\right)$ for each $\left.x \in H\right\}$.

It is easy to see that the power $\mathbf{G}^{\mathbf{H}}$ of $n$-ary hyperalgebras is idempotent whenever $\mathbf{G}$ is idempotent (and medial).

Proposition 2. Let $\mathbf{G}_{i}, i \in I$, be a nonempty family of medial $n$-ary hyperalgebras and $\mathbf{H}$ be an n-ary hyperalgebra. If $\bigotimes_{i \in I} \mathbf{G}_{i}$ is medial, then

$$
\bigotimes_{i \in I} \mathbf{G}_{\mathbf{i}}^{\mathbf{H}} \preceq\left(\bigotimes_{\mathbf{i} \in \mathbf{I}} \mathbf{G}_{\mathbf{i}}\right)^{\mathbf{H}}
$$

Proof. Let $\mathbf{G}_{i}=\left(G_{i}, p^{i}\right)$ for every $i \in I, \mathbf{H}=(H, q), \bigotimes_{i \in I} \mathbf{G}_{\mathbf{i}}=\left(\prod_{i \in I} G_{i}, r\right)$, $\mathbf{G}_{\mathbf{i}}^{\mathbf{H}}=\left(\operatorname{Hom}\left(\mathbf{H}, \mathbf{G}_{\mathbf{i}}\right), u^{i}\right)$ for every $i \in I, \bigotimes_{i \in I} \mathbf{G}_{\mathbf{i}}^{\mathbf{H}}=\left(\prod_{i \in I} \operatorname{Hom}\left(\mathbf{H}, \mathbf{G}_{\mathbf{i}}\right), s\right)$ and 
$\left(\bigotimes_{i \in I} \mathbf{G}_{\mathbf{i}}\right)^{\mathbf{H}}=\left(\operatorname{Hom}\left(\mathbf{H}, \bigotimes_{i \in I} \mathbf{G}_{\mathbf{i}}\right), t\right)$. We define a map $\alpha: \prod_{i \in I} \operatorname{Hom}(\mathbf{H}$, $\left.\mathbf{G}_{\mathbf{i}}\right) \rightarrow\left(\prod_{i \in I} G_{i}\right)^{H}$ by $\alpha\left(f^{i} ; i \in I\right)(z)=\left(f^{i}(z) ; i \in I\right)$ for each $z \in H$.

Let $\left(f^{i} ; i \in I\right) \in \prod_{i \in I} \operatorname{Hom}\left(\mathbf{H}, \mathbf{G}_{\mathbf{i}}\right)$. Let $h \in \alpha\left(f^{i} ; i \in I\right)\left(q\left(x_{1}, \ldots, x_{n}\right)\right)$. Then $h=\alpha\left(f^{i} ; i \in I\right)(x)$ where $x \in q\left(x_{1}, \ldots, x_{n}\right)$. Thus, $h=\alpha\left(f^{i} ; i \in I\right)(x)=$ $\left(f^{i}(x) ; i \in I\right)$. Since $x \in q\left(x_{1}, \ldots, x_{n}\right)$ and $f^{i} \in \operatorname{Hom}\left(\mathbf{H}, \mathbf{G}_{\mathbf{i}}\right)$ for every $i \in I$, we have $f^{i}(x) \in p^{i}\left(f^{i}\left(x_{1}\right), \ldots, f^{i}\left(x_{n}\right)\right)$ for every $i \in I$. Thus, $h=\left(f^{i}(x) ; i \in I\right) \in$ $r\left(\left(f^{i}\left(x_{1}\right) ; i \in I\right), \ldots,\left(f^{i}\left(x_{n}\right) ; i \in I\right)\right)=r\left(\alpha\left(f^{i} ; i \in I\right)\left(x_{1}\right), \ldots, \alpha\left(f^{i} ; i \in I\right)\left(x_{n}\right)\right)$. Consequently, $\alpha\left(f^{i} ; i \in I\right)\left(q\left(x_{1}, \ldots, x_{n}\right)\right) \subseteq r\left(\alpha\left(f^{i} ; i \in I\right)\left(x_{1}\right), \ldots, \alpha\left(f^{i} ; i \in\right.\right.$ $\left.I)\left(x_{n}\right)\right)$. Therefore, $\alpha\left(f^{i} ; i \in I\right) \in \operatorname{Hom}\left(\mathbf{H}, \bigotimes_{i \in I} \mathbf{G}_{\mathbf{i}}\right)$. We have shown that $\alpha$ maps $\prod_{i \in I} \operatorname{Hom}\left(\mathbf{H}, \mathbf{G}_{\mathbf{i}}\right)$ into $\operatorname{Hom}\left(\mathbf{H}, \bigotimes_{i \in I} \mathbf{G}_{\mathbf{i}}\right)$.

Suppose that $\alpha\left(f^{i} ; i \in I\right)=\alpha\left(g^{i} ; i \in I\right)$ where $\left(f^{i} ; i \in I\right),\left(g^{i} ; i \in I\right) \in$ $\prod_{i \in I} \operatorname{Hom}\left(\mathbf{H}, \mathbf{G}_{\mathbf{i}}\right)$. Then $\left(f^{i}(x) ; i \in I\right)=\alpha\left(f^{i} ; i \in I\right)(x)=\alpha\left(g^{i} ; i \in I\right)(x)=$ $\left(g^{i}(x) ; i \in I\right)$ for every $x \in H$. Therefore, $f^{i}(x)=g^{i}(x)$ for every $i \in I$ and for every $x \in H$. Hence, $f^{i}=g^{i}$ for every $i \in I$. Thus, $\alpha$ : $\prod_{i \in I} \operatorname{Hom}\left(\mathbf{H}, \mathbf{G}_{\mathbf{i}}\right) \rightarrow$ $\operatorname{Hom}\left(\mathbf{H}, \bigotimes_{i \in I} \mathbf{G}_{\mathbf{i}}\right)$ is an injection.

Finally, let $\left(f_{1}^{i} ; i \in I\right), \ldots,\left(f_{n}^{i} ; i \in I\right) \in \prod_{i \in I} \operatorname{Hom}\left(\mathbf{H}, \mathbf{G}_{\mathbf{i}}\right)$. We are to show that $\alpha\left(s\left(\left(f_{1}^{i} ; i \in I\right), \ldots,\left(f_{n}^{i} ; i \in I\right)\right)\right)=t\left(\alpha\left(f_{1}^{i} ; i \in I\right), \ldots, \alpha\left(f_{n}^{i} ; i \in I\right)\right)$. It is easy to see that the following seven conditions are equivalent:

(a) $f \in \alpha\left(s_{\lambda}\left(\left(f_{1}^{i} ; i \in I\right), \ldots,\left(f_{n}^{i} ; i \in I\right)\right)\right)$;

(b) $f=\alpha\left(f^{i} ; i \in I\right)$ where $\left(f^{i} ; i \in I\right) \in s\left(\left(f_{1}^{i} ; i \in I\right), \ldots,\left(f_{n}^{i} ; i \in I\right)\right)$;

(c) $f=\alpha\left(f^{i} ; i \in I\right)$ and there exists $J \subseteq I$, card $J \leq 1$, such that $f^{i} \in$ $u^{i}\left(f_{1}^{i}, \ldots, f_{n}^{i}\right)$ for every $i \in I \backslash J$ and $f^{i}=f_{1}^{i}=f_{2}^{i}=\ldots=f_{n}^{i}$ for every $i \in J$

(d) $f(x)=\left(f^{i}(x) ; i \in I\right)$ for every $x \in H$ and there exists $J \subseteq I$, card $J \leq 1$, such that $f^{i}(x) \in p_{\lambda}^{i}\left(f_{1}^{i}(x), \ldots, f_{n}^{i}(x)\right)$ for every $i \in I \backslash J$ and every $x \in H$ and $f^{i}(x)=f_{1}^{i}(x)=f_{2}^{i}(x)=\ldots=f_{n}^{i}(x)$ for every $i \in J$ and every $x \in H$;

(e) $f(x)=\left(f^{i}(x) ; i \in I\right) \in r\left(\left(f_{1}^{i}(x) ; i \in I\right), \ldots,\left(f_{n}^{i}(x) ; i \in I\right)\right)$ for every $x \in$ $H$

(f) $f(x) \in r\left(\alpha\left(f_{1}^{i} ; i \in I\right)(x), \ldots, \alpha\left(f_{n}^{i} ; i \in I\right)(x)\right)$ for every $x \in H$;

(g) $f \in t\left(\alpha\left(f_{1}^{i} ; i \in I\right), \ldots, \alpha\left(f_{n}^{i} ; i \in I\right)\right)$.

Consequently, $\alpha\left(s\left(\left(f_{1}^{i} ; i \in I\right), \ldots,\left(f_{n}^{i} ; i \in I\right)\right)\right)=t\left(\alpha\left(f_{1}^{i} ; i \in I\right), \ldots, \alpha\left(f_{n}^{i} ; i \in\right.\right.$ $I))$, which yields $\bigotimes_{i \in I} \mathbf{G}_{\mathbf{i}}^{\mathbf{H}} \preceq\left(\bigotimes_{\mathbf{i} \in \mathbf{I}} \mathbf{G}_{\mathbf{i}}\right)^{\mathbf{H}}$.

Remark 2. It may easily be shown that, in the previous Proposition, we may write $\cong$ instead of $\preceq$ provided that $\mathbf{G}_{\mathbf{i}}$ is idempotent for every $i \in I$. We then obtain the so-called second exponential law for the cartesian product, i.e., the law $\prod_{i \in I} \mathbf{G}_{\mathbf{i}}^{\mathbf{H}} \cong$ $\left(\prod_{\mathbf{i} \in \mathbf{I}} \mathbf{G}_{\mathbf{i}}\right)^{\mathbf{H}}$.

Theorem 2. Let $\mathbf{G}, \mathbf{H}$ be n-ary hyperalgebras. If $\mathbf{G}$ is medial, then so is $\mathbf{G}^{\mathbf{H}}$.

Proof. Let $\mathbf{H}=(H, q), \mathbf{G}=(G, p), \mathbf{G}^{\mathbf{H}}=(\operatorname{Hom}(\mathbf{H}, \mathbf{G}), r)$ and let $\left(f_{i j}\right)$ be an $n \times n$-matrix over $\operatorname{Hom}(\mathbf{H}, \mathbf{G})$. Suppose that $h_{i} \in r\left(f_{i 1}, \ldots, f_{i n}\right)$ for all $i=1, \ldots, n$ 
and $g_{j} \in r\left(f_{1 j}, \ldots, f_{n j}\right)$ for all $j=1, \ldots, n$. For every $x \in H$, we have $h_{i}(x) \in$ $p\left(f_{i 1}(x), \ldots, f_{i n}(x)\right)$ for all $i=1, \ldots, n$ and $g_{j}(x) \in p\left(f_{1 j}(x), \ldots, f_{n j}(x)\right)$ for all $j=1, \ldots, n$. Since $\mathbf{G}$ is medial, we have $p\left(h_{1}(x), \ldots, h_{n}(x)\right)=p\left(g_{1}(x), \ldots, g_{n}(x)\right)$ for every $x \in H$. Therefore, $r\left(h_{1}, \ldots, h_{n}\right)=r\left(g_{1}, \ldots, g_{n}\right)$, so that $\mathbf{G}^{\mathbf{H}}$ is medial.

Definition 3. An $n$-ary hyperalgebra $(G, p)$ is called diagonal if, for every $n \times n$ matrix $\left(a_{i j}\right)$ over $G$, we have $p\left(p\left(a_{11}, \ldots, a_{1 n}\right), \ldots, p\left(a_{n 1}, \ldots, a_{n n}\right)\right) \cap$ $p\left(p\left(a_{11}, \ldots, a_{n 1}\right), \ldots, p\left(a_{1 n}, \ldots, a_{n n}\right)\right) \subseteq p\left(a_{11}, \ldots, a_{n n}\right)$.

For idempotent algebras, the diagonality introduced coincides with the diagonality studied in [9].

Example 2. It may easily be seen that the hypergroupoid from Example 1(1) is diagonal.

The combined product of diagonal $n$-ary hyperalgebras is not be diagonal in general. But we have:

Proposition 3. Let $\mathbf{G}, \mathbf{H}$ be n-ary hyperalgebras. If $\overline{\mathbf{G}}, \overline{\mathbf{H}}$ are diagonal, then so is $\overline{\mathbf{G} \otimes \mathbf{H}}$.

Proof. The proof is analogous to that of Proposition 1.

Theorem 3. Let $\mathbf{G}, \mathbf{H}$ be n-ary hyperalgebras. If $\mathbf{G}$ is medial and diagonal, then $\mathbf{G}^{\mathbf{H}}$ is diagonal.

Proof. Let $\mathbf{H}=(H, q), \mathbf{G}=(G, p), \mathbf{G}^{\mathbf{H}}=(\operatorname{Hom}(\mathbf{H}, \mathbf{G}), r)$ and let $\left(f_{i j}\right)$ be an $n \times$ $n$-matrix over $\operatorname{Hom}(\mathbf{H}, \mathbf{G})$. Suppose that $f \in r\left(r\left(f_{11}, \ldots, f_{1 n}\right), \ldots, r\left(f_{n 1}\right.\right.$, $\left.\left.\ldots, f_{n n}\right)\right) \cap r\left(r\left(f_{11}, \ldots, f_{n 1}\right), \ldots, r\left(f_{1 n}, \ldots, f_{n n}\right)\right)$. Then $f(x) \in p\left(p\left(f_{11}(x), \ldots\right.\right.$, $\left.\left.f_{1 n}(x)\right), \ldots, r\left(f_{n 1}(x), \ldots, f_{n n}(x)\right)\right) \cap p\left(p\left(f_{11}(x), \ldots, f_{n 1}(x)\right), \ldots, p\left(f_{1 n}(x), \ldots\right.\right.$, $\left.\left.f_{n n}(x)\right)\right)$ for every $x \in H$. Since $\mathbf{G}$ is diagonal, we have $f(x) \in p\left(f_{11}(x), \ldots\right.$, $\left.f_{n n}(x)\right)$ for every $x \in H$. Thus, $f \in r\left(f_{11}, \ldots, f_{n n}\right)$. Hence, $\mathbf{G}^{\mathbf{H}}$ is diagonal.

We denote by $M D H Y P_{n}$ the full subconstruct of $H Y P_{n}$ whose objects are the $n$-ary hyperalgebras that are medial and diagonal.

Lemma 2. Let $\mathbf{G} \in M D H Y P_{n}$ and $\mathbf{H}, \mathbf{K} \in H Y P_{n}$. Then the canonical bijection $\varphi:\left(G^{H}\right)^{K} \rightarrow G^{H \times K}$ restricted to $\operatorname{Hom}\left(\mathbf{K}, \mathbf{G}^{\mathbf{H}}\right)$ is a bijection of Hom $\left(\mathbf{K}, \mathbf{G}^{\mathbf{H}}\right)$ onto $\operatorname{Hom}(\mathbf{H} \otimes \mathbf{K}, \mathbf{G})$.

Proof. Let $\mathbf{H}=(H, q), \mathbf{G}=(G, p), \mathbf{K}=(K, s), \mathbf{G}^{\mathbf{H}}=(\operatorname{Hom}(\mathbf{H}, \mathbf{G}), r), \mathbf{H} \otimes \mathbf{K}=$ $(H \times K, v)$ and let $\mathbf{G}$ be diagonal and medial. Let $h \in \operatorname{Hom}\left(\mathbf{K}, \mathbf{G}^{\mathbf{H}}\right)$ and let $\left(y_{1}, z_{1}\right), \ldots,\left(y_{n}, z_{n}\right) \in H \times K$. To show that $\varphi(h)\left(v\left(\left(y_{1}, z_{1}\right), \ldots,\left(y_{n}, z_{n}\right)\right) \subseteq\right.$ $p\left(\varphi(h)\left(y_{1}, z_{1}\right), \ldots, \varphi(h)\left(y_{n}, z_{n}\right)\right)$, let $x \in \varphi(h)\left(v\left(\left(y_{1}, z_{1}\right), \ldots,\left(y_{n}, z_{n}\right)\right)\right)$. Then there exists $(y, z) \in v\left(\left(y_{1}, z_{1}\right), \ldots,\left(y_{n}, z_{n}\right)\right)$ such that $x=\varphi(h)(y, z)$. Thus, one of the following three conditions is satisfied:

(i) $y \in q\left(y_{1}, \ldots, y_{n}\right)$ and $z \in s\left(z_{1}, \ldots, z_{n}\right)$, 
(ii) $y=y_{1}=y_{2}=\ldots=y_{n}$ and $z \in s\left(z_{1}, \ldots, z_{n}\right)$,

(iii) $y \in q\left(y_{1}, \ldots, y_{n}\right)$ and $z=z_{1}=z_{2}=\ldots=z_{n}$.

If $z \in s\left(z_{1}, \ldots, z_{n}\right)$, then $h(z) \in h\left(s\left(z_{1}, \ldots, z_{n}\right)\right) \subseteq r\left(h\left(z_{1}\right), \ldots, h\left(z_{n}\right)\right)$, hence $\varphi(h)(y, z)=h(z)(y) \in p\left(\left(h\left(z_{1}\right)(y), \ldots, h\left(z_{n}\right)(y)\right)=p\left(\varphi(h)\left(y, z_{1}\right), \ldots\right.\right.$, $\varphi(h)\left(y, z_{n}\right)$ for each $y \in H$. Thus, $\varphi(h)(y, z) \in p\left(\varphi(h)\left(y_{1}, z_{1}\right), \ldots, \varphi(h)\left(y_{n}, z_{n}\right)\right)$ provided that (ii) is satisfied.

If $y \in q\left(y_{1}, \ldots, y_{n}\right)$, then $\varphi(h)(y, z)=h(z)(y) \in h(z)\left(q\left(y_{1}, \ldots, y_{n}\right)\right) \subseteq$ $p\left(h(z)\left(y_{1}\right), \ldots, h(z)\left(y_{n}\right)\right)=p\left(\varphi(h)\left(y_{1}, z\right), \ldots, \varphi(h)\left(y_{n}, z\right)\right)$ for every $z \in K$. Thus, $\varphi(h)(y, z) \in p\left(\varphi(h)\left(y_{1}, z_{1}\right), \ldots, \varphi(h)\left(y_{n}, z_{n}\right)\right)$ provided that (iii) is satisfied.

Suppose that (i) is satisfied. Since $z \in s\left(z_{1}, \ldots, z_{n}\right)$, by the above considerations we get $\varphi(h)\left(y_{i}, z\right) \in p\left(\varphi(h)\left(y_{i}, z_{1}\right), \ldots, \varphi(h)\left(y_{i}, z_{n}\right)\right)$ for every $i=1, \ldots, n$ and $\varphi(h)(y, z) \in p\left(\varphi(h)\left(y, z_{1}\right), \ldots, \varphi(h)\left(y, z_{n}\right)\right)$. As $y \in q\left(y_{1}, \ldots, y_{n}\right)$, by the above considerations we obtain $\varphi(h)\left(y, z_{j}\right) \in p\left(\varphi(h)\left(y_{1}, z_{j}\right), \ldots, \varphi(h)\left(y_{n}, z_{j}\right)\right)$ for every $j=1, \ldots, n$ and $\varphi(h)(y, z) \in p\left(\varphi(h)\left(y_{1}, z\right), \ldots, \varphi(h)\left(y_{n}, z\right)\right)$. The diagonality of $\mathbf{G}$ implies $\varphi(h)(y, z) \in p\left(\varphi(h)\left(y_{1}, z_{1}\right), \ldots, \varphi(h)\left(y_{n}, z_{n}\right)\right)$. We have shown that $\varphi(h) \in$ $\operatorname{Hom}(\mathbf{H} \otimes \mathbf{K}, \mathbf{G})$.

Let $g \in \operatorname{Hom}(\mathbf{H} \otimes \mathbf{K}, \mathbf{G})$ and $z_{1}, \ldots, z_{n} \in K$. To show that $\varphi^{-1}(g)\left(s\left(z_{1}, \ldots\right.\right.$, $\left.\left.z_{n}\right)\right) \subseteq r\left(\varphi^{-1}(g)\left(z_{1}\right), \ldots, \varphi^{-1}(g)\left(z_{n}\right)\right)$, let $x \in \varphi^{-1}(g)\left(s\left(z_{1}, \ldots, z_{n}\right)\right)$. Then there exists $z \in s\left(z_{1}, \ldots, z_{n}\right)$ such that $x \in \varphi^{-1}(g)(z)$. Let $y \in H$. Then $(y, z) \in$ $v\left(\left(y, z_{1}\right), \ldots,\left(y, z_{n}\right)\right)$. Since $g \in \operatorname{Hom}(\mathbf{H} \otimes \mathbf{K}, \mathbf{G})$, we have $g(y, z) \in g\left(v\left(\left(y, z_{1}\right)\right.\right.$ $\left., \ldots,\left(y, z_{n}\right)\right) \subseteq p\left(g\left(y, z_{1}\right), \ldots, g\left(y, z_{n}\right)\right)$. It follows that $\varphi^{-1}(g)(z)(y) \in p\left(\varphi^{-1}(g)\right.$ $\left.\left(z_{1}\right)(y), \ldots, \varphi^{-1}(g)\left(z_{n}\right)(y)\right)$, hence $\varphi^{-1}(g)(z) \in p\left(\varphi^{-1}(g)\left(z_{1}\right), \ldots, \varphi^{-1}(g)\left(z_{n}\right)\right)$. Therefore, $\varphi^{-1}(g)\left(s\left(z_{1}, \ldots, z_{n}\right)\right) \subseteq r\left(\varphi^{-1}(g)\left(z_{1}\right), \ldots, \varphi^{-1}(g)\left(z_{n}\right)\right)$. Consequently, $\varphi^{-1}(g) \in \operatorname{Hom}\left(\mathbf{K}, \mathbf{G}^{\mathbf{H}}\right)$.

Given $\mathbf{G} \in M D H Y P_{n}$ and $\mathbf{H}=(H, q) \in H Y P_{n}$, the evaluation map is the map $e: \mathbf{H} \otimes \mathbf{G}^{\mathbf{H}} \rightarrow \mathbf{G}$ defined by $e(y, f)=f(y)$ whenever $y \in H$ and $f \in \operatorname{Hom}(\mathbf{H}, \mathbf{G})$.

Theorem 4. Let $\mathbf{G} \in M D H Y P_{n}$ and $\mathbf{H} \in H Y P_{n}$. Then the pair $\left(\mathbf{G}^{\mathbf{H}}, e\right)$, where $e: \mathbf{H} \otimes \mathbf{G}^{\mathbf{H}}$ is the evaluation map, is a co-universal arrow for $\mathbf{G}$ with respect to the functor $\mathbf{H} \otimes-: H Y P_{n} \rightarrow H Y P_{n}$.

Proof. Let $\mathbf{G}=(G, p), \mathbf{H}=(H, q)$ and $\mathbf{H} \otimes \mathbf{G}^{\mathbf{H}}=(H \times H o m(\mathbf{H}, \mathbf{G}), w)$. For every $\mathbf{K}=(K, r) \in H Y P_{n}$ and every morphism $f: \mathbf{H} \otimes \mathbf{K} \rightarrow \mathbf{G}$, the map $f^{*}: \mathbf{K} \rightarrow$ $\mathbf{G}^{\mathbf{H}}$ given by $f^{*}(z)(y)=f(y, z)$ is a morphism in $H Y P_{n}$ by the previous Lemma. We will show that the evaluation map $e: \mathbf{H} \otimes \mathbf{G}^{\mathbf{H}} \rightarrow \mathbf{G}$ is morphism in $H Y P_{n}$, too. To this end, let $x \in e\left(w\left(\left(y_{1}, h_{1}\right), \ldots,\left(y_{n}, h_{n}\right)\right)\right)$. Then $x=e(y, h)=h(y)$ where $(y, h) \in w\left(\left(y_{1}, h_{1}\right), \ldots,\left(y_{n}, h_{n}\right)\right)$. Thus, we have

(i) $y \in q\left(y_{1}, \ldots, y_{n}\right)$ and $h \in r\left(h_{1}, \ldots, h_{n}\right)$ or

(ii) $y=y_{1}=y_{2}=\ldots=y_{n}$ and $h \in r\left(h_{1}, \ldots, h_{n}\right)$ or

(iii) $y \in q\left(y_{1}, \ldots, y_{n}\right)$ and $h=h_{1}=h_{2}=\ldots=h_{n}$.

Suppose that (i) is satisfied. Then $h(y) \in p\left(h\left(y_{1}\right), \ldots, h\left(y_{n}\right)\right)$ and $h\left(y_{i}\right) \in$ $p\left(h_{1}\left(y_{i}\right), \ldots, h_{n}\left(y_{i}\right)\right)$ for every $i=1, \ldots, n$. Thus, we have $x=h(y) \in$ 
$p\left(p\left(h_{1}\left(y_{1}\right), \ldots, h_{n}\left(y_{1}\right)\right), \ldots, p\left(h_{1}\left(y_{n}\right), \ldots, h_{n}\left(y_{n}\right)\right)\right)$. Further, we have $h(y) \in$ $p\left(h_{1}(y), \ldots, h_{n}(y)\right)$ and $h_{j}(y) \in p\left(h_{j}\left(y_{1}\right), \ldots, h_{j}\left(y_{n}\right)\right)$ for every $j=1, \ldots, n$. Hence, $h(y) \in p\left(p\left(h_{1}\left(y_{1}\right), \ldots, h_{1}\left(y_{n}\right)\right), \ldots, p\left(h_{n}\left(y_{1}\right), \ldots, h_{n}\left(y_{n}\right)\right)\right)$. Since $\mathbf{G}$ is diagonal, we have $x \in p\left(h_{1}\left(y_{1}\right), \ldots, h_{n}\left(y_{n}\right)\right)=p\left(e\left(y_{1}, h_{1}\right), \ldots, e\left(y_{n}, h_{n}\right)\right)$. Therefore, $e: \mathbf{H} \otimes \mathbf{G}^{\mathbf{H}} \rightarrow \mathbf{G}$ is a homomorphism.

Suppose that (ii) is true. Then $h(y)=h\left(y_{1}\right)=\ldots=h\left(y_{n}\right)$ and $h\left(y_{i}\right) \in$ $p\left(h_{1}\left(y_{i}\right), \ldots, h_{n}\left(y_{i}\right)\right)$ for every $i=1, \ldots, n$. Thus, we have $x=h(y) \in$ $p\left(h_{1}(y), \ldots, h_{n}(y)\right)=p\left(e\left(y_{1}, h_{1}\right), \ldots, e\left(y_{n}, h_{n}\right)\right)$. Therefore, $e: \mathbf{H} \otimes \mathbf{G}^{\mathbf{H}} \rightarrow \mathbf{G}$ is a homomorphism.

Finally, let (iii) be true. Then $(h) \in p\left(h\left(y_{1}\right), \ldots, h\left(y_{n}\right)\right)$ and $h\left(y_{i}\right)=h_{1}\left(y_{i}\right)=$ $\ldots=h_{n}\left(y_{i}\right)$ for every $i=1, \ldots, n$. Thus, we have $x=h(y) \in p\left(h\left(y_{1}\right), \ldots, h\left(y_{n}\right)\right)=$ $p\left(e\left(y_{1}, h_{1}\right), \ldots, e\left(y_{n}, h_{n}\right)\right)$. Therefore, $e: \mathbf{H} \otimes \mathbf{G}^{\mathbf{H}} \rightarrow \mathbf{G}$ is a homomorphism.

Remark 3. The full subconstruct $M D H Y P_{n}$ of $H Y P_{n}$ is closed under powers but it is not closed under combined products. By the previous Theorem, for every full subconstruct $\mathcal{A}$ of $M D H Y P_{n}$ closed under both the powers and combined products, and every $\mathcal{A}$-object $\mathbf{H}$, the functor $\mathbf{H} \otimes-: \mathcal{A} \rightarrow \mathcal{A}$ is a co-adjoint with the corresponding adjoint being the functor $-\mathbf{H}: \mathcal{A} \rightarrow \mathcal{A}$. But it follows from [14] that the functor $-{ }^{\mathbf{H}}: \mathcal{A} \rightarrow \mathcal{A}$ is an adjoint with the corresponding co-adjoint being the functor $\mathbf{H} \times-: \mathcal{A} \rightarrow \mathcal{A}$. Consequently, the functors $\mathbf{H} \otimes-: \mathcal{A} \rightarrow \mathcal{A}$ and $\mathbf{H} \times-: \mathcal{A} \rightarrow \mathcal{A}$ are naturally isomorphic and $\mathcal{A}$ is cartesian closed. Therefore, all objects of $\mathcal{A}$ have to be idempotent. Such a subconstruct $\mathcal{A}$ may be obtained by putting $\mathcal{A}=I M D H Y P_{n}$ where $I M D H Y P_{n}$ is the full subconstruct of $M D H Y P_{n}$ given by its idempotent objects. It follows from [14] that IMDHY $P_{n}$ is cartesian closed.

Proposition 4. Let $\mathbf{G}, \mathbf{H}, \mathbf{K}$ be n-ary hyperalgebras. If $\mathbf{G}$ is medial and diagonal, then

$$
\left(\mathbf{G}^{\mathbf{H}}\right)^{\mathbf{K}} \cong \mathbf{G}^{\mathbf{H} \otimes \mathbf{K}} .
$$

Proof. Let $\mathbf{H}=(H, q), \mathbf{G}=(G, p), \mathbf{K}=(K, s), \mathbf{G}^{\mathbf{H}}=(\operatorname{Hom}(\mathbf{H}, \mathbf{G}), r), \mathbf{H} \otimes \mathbf{K}=$ $(H \times K, v),\left(\mathbf{G}^{\mathbf{H}}\right)^{\mathbf{K}}=\left(\operatorname{Hom}\left(\mathbf{K}, \mathbf{G}^{\mathbf{H}}\right), t\right), \mathbf{G}^{\mathbf{H} \otimes \mathbf{K}}=(\operatorname{Hom}(\mathbf{H} \otimes \mathbf{K}, \mathbf{G}), u)$ and let $\mathbf{G}$ be diagonal and medial.

Let $h_{1}, \ldots, h_{n} \in \operatorname{Hom}\left(\mathbf{K}, \mathbf{G}^{\mathbf{H}}\right)$ and $x \in \varphi\left(t\left(h_{1}, \ldots, h_{n}\right)\right)$. Then there exists $h \in$ $t\left(h_{1}, \ldots, h_{n}\right)$ such that $x=\varphi(h)$. Since $h \in t\left(h_{1}, \ldots, h_{n}\right)$, we have $h(z) \in$ $r\left(h_{1}(z), \ldots, h_{n}(z)\right)$ for every $z \in K$. Therefore, $h(z)(y) \in p\left(h_{1}(z)(y), \ldots, h_{n}(z)(y)\right)$ for eacu $y \in H$. Thus, $\varphi(h)(y, z) \in p\left(\varphi\left(h_{1}\right)(y, z), \ldots, \varphi\left(h_{n}\right)(y, z)\right)$ for every $(y, z) \in$ $H \times K$. Hence, $\varphi(h) \in u\left(\varphi\left(h_{1}\right), \ldots, \varphi\left(h_{n}\right)\right)$. Consequently, $\varphi\left(t\left(h_{1}, \ldots, h_{n}\right)\right) \subseteq$ $u\left(\varphi\left(h_{1}\right), \ldots, \varphi\left(h_{n}\right)\right)$.

Conversely, let $x \in u\left(\varphi\left(h_{1}\right), \ldots, \varphi\left(h_{n}\right)\right)$. By Lemma 2, there exists $h \in$ $\operatorname{Hom}\left(\mathbf{K}, \mathbf{G}^{\mathbf{H}}\right)$ with $x=\varphi(h)$, so that $\varphi(h) \in u\left(\varphi\left(h_{1}\right), \ldots, \varphi\left(h_{n}\right)\right)$. Then $\varphi(h)(y, z) \in$ $p\left(\varphi\left(h_{1}\right)(y, z), \ldots, \varphi\left(h_{n}\right)(y, z)\right)$ for every $(y, z) \in H \times K$, which implies $h(z)(y) \in$ $p\left(h_{1}(z)(y), \ldots, h_{n}(z)(y)\right)$ for every $y \in H$ and $z \in K$. It follows that $h(z) \in$ 
$r\left(h_{1}(z), \ldots, h_{n}(z)\right)$ for every $z \in K$. Therefore, $h \in t\left(h_{1}, \ldots, h_{n}\right)$, thus $\varphi(h) \in$ $\varphi\left(t\left(h_{1}, \ldots, h_{n}\right)\right)$. Hence, $u\left(\varphi\left(h_{1}\right), \ldots, \varphi\left(h_{n}\right)\right) \subseteq \varphi\left(t\left(h_{1}, \ldots, h_{n}\right)\right)$.

We have shown that $\varphi\left(t\left(h_{1}, \ldots, h_{n}\right)\right)=u\left(\varphi\left(h_{1}\right), \ldots, \varphi\left(h_{n}\right)\right)$ whenever $h_{1}$, $\ldots, h_{n} \in \operatorname{Hom}\left(\mathbf{K}, \mathbf{G}^{\mathbf{H}}\right)$. Thus, $\left(\mathbf{G}^{\mathbf{H}}\right)^{\mathbf{K}}$ is isomorphic to $\mathbf{G}^{\mathbf{H} \otimes \mathbf{K}}$ by Lemma 2.

Remark 4. It follows from Proposition 4 that the exponential law $\left(\mathbf{G}^{\mathbf{H}}\right)^{\mathbf{K}} \cong \mathbf{G}^{\mathbf{H} \times \mathbf{K}}$ is valid whenever $\mathbf{G}, \mathbf{H}, \mathbf{K}$ are $n$-ary hyperalgebras, $\mathbf{G}$ medial and diagonal and $\mathbf{H}, \mathbf{K}$ idempotent. This result follows from [4]. But Proposition 4 says that, when replacing the cartesian product by the combined one, the above exponential law is satisfied for any $n$-ary hyperalgebras $\mathbf{H}, \mathbf{K}$, not only for the idempotent ones.

\section{REFERENCES}

[1] J. Adámek, H. Herrlich, and G. Strecker, Abstract and Concrete Categories. New York: Wiley \& Sons, 1990

[2] G. Birkhoff, "Generalized arithmetic," Duke Math. J., vol. 9, pp. 283-302, 1942.

[3] P. Corsini, Algebraic Hyperstructures and Applications, ser. Proceedings of the Sixth International Congress, Prague, 1996. Greece: Democritus University of Thrace, 1997.

[4] J. Ježek and T. Kepka, Medial groupoids, ser. Rozpravy ČSAV, Ǩada Mat. a Přír. Věd. 93/1. Prague: Academia, 1983.

[5] L. Klukovits, "On commutative universal algebras," Acta Sci. Math. (Szeged), vol. 34, pp. 171174, 1973.

[6] F. Linton, "Autonomous equational categories," J. Math. Mech., vol. 15, pp. 637-642, 66.

[7] V. Novák, "On a power of relational structures," Czech. Math. J., vol. 35, 1985.

[8] M. Novotný and J. Šlapal, "On powers of relational structures," Publ. Math. Debrecen, vol. 61, pp. 341-356, 2002.

[9] J. Plonka, "Diagonal algebras," Fund. Math., vol. 58, pp. 309-321, 1966.

[10] J. Šlapal, "Direct arithmetic of relational systems," Publ. Math. Debrecen, vol. 38, pp. 39-48, 1991.

[11] J. Šlapal, "Cardinal arithmetic of general relational systems," Czech. Math. J., vol. 43, pp. 125139, 1993.

[12] J. Šlapal, "A cartesian closed category of universal algebras," Acta Sci. Math. (Szeged), vol. 58, pp. 93-97, 1993.

[13] J. Šlapal, “On exponentiation of $n$-ary algebras,” Acta Math. Hungar., vol. 63, pp. 313-322, 1994.

[14] J. Šlapal, “On exponentiation of universal hyperalgebras," Alg. Universalis, vol. 44, pp. 187-193, 2000.

Author's address

J. Šlapal

Brno University of Technology, Institute of Mathematics, Technická 2, 61669 Brno, Czech Republic

E-mail address: slapal@ fme.vutbr.cz 